\title{
Chinese Teenager Physical Health Information Service System Construc- tion Research
}

\author{
Yuefeng Zhao*
}

China Department of Public Instruction, Wenzhou Vocational and Technical College, Wenzhou 325035, China

\begin{abstract}
The paper's research on Chinese teenager physical health information service system construction targeted at teenagers, it mainly involves objective selection, classification and sorting of selected objectives as well as endowing weights on all indicators, synthesizing these three aspects, the paper carries on type test according to fuzzy clustering and combining with specific examples that takes normalization processing with multiple indicators, we further get fuzzy matrix weight coefficient, and obtain paper's selected four schoolgirls physique accurate result is that No.1 is 36 , No.2 is 49.8 , No.3 is 61.7 while No.4 is 64.6 through the method of fuzzy aggregation, from which it is clear about every schoolgirl physical conditions, so it proves the model's validity and rationality.
\end{abstract}

Keywords: Fuzzy clustering, information service, physical health, teenager.

\section{INTRODUCTION}

Physique refers to human physical quality conditions, it will change with age changes, there are great differences among different ages, period of teenager is the golden age in life, teenager physical conditions not only involve individual personal quality, but also the more important is it has connections with state future and destiny [1], so research on teenager physical health field is of great significance [2].

Regarding physical health field research, formers have made lots of efforts and gained certain achievements, such as Zhang $\mathrm{Yu}$ in Children and teenager physical evaluation, he had ever utilized China's Guangzhou, Xiangtan, Wuhan and Harbin four sites as research objects [3], carried out research on teenager physical health, and made evaluation on them through fuzzy analytic hierarchy process method, finally got that the article mentioned evaluation system was of reality in children and teenager physical evaluation system, and made comparison of different regions' teenagers physical health, got that Xiangtan, Wuhan and Harbin's were inferior to Guangzhou and other regions [4].

By formers researches, the paper combines with formers experiences to make further analysis and research on teenager physical issue, and analyze them by using fuzzy clustering, analytic hierarchy process and other methods, which provides theoretical supports for its exploration in teenager physical health research [5].

\section{TEENAGER PHYSICAL HEALTH INFORMATION SERVICE SYSTEM THEORETICAL MODELS}

Teenager physical health information service system construction, firstly it should select indicators, and classify and sort out them, but limits among them is not obvious, and so the paper applies fuzzy clustering method to handle with them, its principle is as following [6].

In daily life, we tend to need classifying multiple indicators according to certain criterions. But in most cases, classification limits are not very obvious, therefore fuzzy clustering analysis application in practice is very widely [7].

Fuzzy clustering analysis generally has following steps:

Establish matrix

Set classified object is $U=\left\{u_{1}, u_{2}, \nwarrow, u_{n}\right\}$, every object has $m$ pieces of indicators to show its character:

$x_{i}=\left\{x_{i 1}, x_{i 2}, \nwarrow, x_{i n}\right\}, i=1,2, \nwarrow, n$

Therefore it appears matrix:

$X=\left(x_{i j}\right)_{n \times m}$

In real application, different data corresponds to different dimensions, to easier comparing data, it needs to normalize data; in the following it introduces some common normalization methods.

Method that standardized standard deviation:

$x_{i j}^{\prime}=\frac{x_{i j}-\overline{x_{j}}}{s_{j}}, i=1,2, \nwarrow, n ; j=1,2, \nwarrow, m$

Among them:

$\bar{x}_{j}=\frac{1}{n} \sum_{i=1}^{n} x_{i j}, s_{j}=\sqrt{\frac{1}{n} \sum_{i=1}^{n}\left(x_{i j}-\bar{x}_{j}\right)^{2}}$

Range orthonormal method:

$\dot{x}_{i j}^{\prime}=\frac{x_{i j}-\min \left\{x_{k j}\right\}}{\max _{1 \leq k \leq n}\left\{x_{k j}\right\} \min _{1 \leq k \leq n}\left\{k_{b j}\right\}}, i=1,2, \nwarrow, n ; j=1,2, \nwarrow, m$ 
Range standardized method:

$\dot{x}_{i j}^{\prime}=\frac{x_{i j}-x_{j}}{\max _{1 \leq k \leq n}\left\{x_{k j}\right\} \min _{1 \leq k \leq n}\left\{k_{k j}\right\}}, i=1,2, \nwarrow, n ; j=1,2, \nwarrow, m$

Maximum value normalized method:

$\dot{x}_{i j}^{\prime}=\frac{x_{i j}}{\max _{1 \leq k \leq n}\left\{x_{k j}\right\}}, i=1,2, \nwarrow, n ; j=1,2, \nwarrow, m$

Fuzzy similar matrix establishment

Establish $x_{i}$ and $x_{j}$ similarity level $r_{i j}$ generally it has following methods.

Similarity coefficient method includes included angle cosine method and correlation coefficient method.

Included angle cosine method:

$r_{i j}=\frac{\sum_{k=1}^{m} x_{i k} x_{j k}}{\sqrt{\sum_{k=1}^{m} x_{i k}^{2}} \sqrt{\sum_{k=1}^{m} x_{j k}^{2}}}$

Correlation coefficient method:

$$
r_{i j}=\frac{\sum_{k=1}^{m}\left|x_{i k}-\overline{x_{i}}\right|\left|x_{j k}-\overline{x_{j}}\right|}{\sqrt{\sum_{k=1}^{m} x_{i k}^{2}} \sqrt{\sum_{k=1}^{m} x_{j k}^{2}}}
$$

Distance method:

In general, when using distance method, $r_{i j}=1-c\left(d\left(x_{i}, x_{j}\right)\right)^{\alpha}, c, \alpha$ are selected suitable parameters that let $0 \leq r_{i j} \leq 1$ to be true.

The method used distance $d\left(x_{i}, x_{j}\right)$ computational method is as following:

Euclidean distance:

$d\left(x_{i}, x_{j}\right)=\sqrt{\sum_{k=1}^{m}\left(x_{i k}-x_{j k}\right)^{2}}$

Hamming distance:

$d\left(x_{i}, x_{j}\right)=\sum_{k=1}^{m}\left|x_{i k}-x_{j k}\right|$

Chebyshev distance:

$d\left(x_{i}, x_{j}\right)=\max _{1 \leq k \leq m}\left|x_{i k}-x_{j k}\right|$

Close degree method includes geometric average minimum method, maximin method and arithmetic average minimum method, the methods is as following:

Geometric average minimum method:

$$
r_{i j}=\frac{\sum_{k=1}^{m}\left(x_{i k} \wedge x_{j k}\right)}{\sum_{k=1}^{m} \sqrt{x_{i k} x_{j k}}}
$$

Maximin method:

$r_{i j}=\frac{\sum_{k=1}^{m}\left(x_{i k} \wedge x_{j k}\right)}{\sum_{k=1}^{m}\left(x_{i k} \vee x_{j k}\right)}$

Arithmetic average minimum method:

$r_{i j}=\frac{\sum_{k=1}^{m}\left(x_{i k} \wedge x_{j k}\right)}{\frac{1}{2} \sum_{k=1}^{m}\left(x_{i k}+x_{j k}\right)}$

Finally, it solves its fuzzy clustering indicator comprehensive scores.

\subsection{Chinese Teenager Physical Health Information Serv- ice System Researches}

In teenager physical health field research, it mainly takes 800 meters running, standing long jump, weight/height*1000, 50 meters running, sit-up as well as lung capacity/weight these indicators as research objects factors, for these indicators weights in the system of the paper, I define the weights by analytic hierarchy process, during calculation process, use different physiques students differences in all indicators as statistics to do normalization unified handling, and apply centesimal form to comprehensive evaluate the selected objects physical conditions.

The paper selects one province schoolgirls physical test result of Chinese teenager physical investigation in 2005 , from which ratio between weight and height is represented by $x_{1}$, ration between lung capacity and weight is represented by $x_{2} ; 50$ meters sprint is represented by $x_{3}$, standing long jump is represented by $x_{4}$, while sit-up is represented by $x_{5}, 800$ meters running is represented by $x_{6}$. Corresponding all indicators test result is as following Table 1.

\subsection{Clustering Processing with Hundred Schoolgirls Physical Test Table}

For above all indicators $x_{1}, x_{2}, x_{3}, x_{4}, x_{5}$, it applies formula $:[(x-\bar{x}) / x]+5$, for above $x_{3}, x_{6}$ speed indicators, it applies formula $5-(x-\bar{x}) / S$ to calculate, and can get all indicators standardized values as following Table 2.

By above Table 2 all indicators variables values being below 5, then it can assume the paper selected sample clustering center is in the level of relative poor physique, samples' indicators variables values mean values are above 5 and then let the class II to represent better physique level and use it as its clustering center, we can adopt formula to do clustering:

$d=\sum_{i=1}^{m}\left|x_{i}-\overline{x_{i}}\right|$ 
Table 1. Hundred schoolgirls physical test table.

\begin{tabular}{|c|c|c|c|c|c|c|}
\hline$n$ & $x_{1}$ & $x_{2}$ & $x_{3}$ & $x_{4}$ & $x_{5}$ & $x_{6}$ \\
\hline 1 & 284.7 & 80.7 & 8.5 & 208 & 36 & 225.4 \\
\hline 2 & 350.16 & 50.5 & 9.3 & 167 & 29 & 239.8 \\
\hline 3 & 365.76 & 59.76 & 9.6 & 153 & 12 & 245.8 \\
\hline$\equiv$ & $\equiv$ & $\equiv$ & $\equiv$ & $\equiv$ & $\equiv$ & $\equiv$ \\
\hline 9 & 312.6 & 45.3 & 9.4 & 160 & 15 & 261.8 \\
\hline$\equiv$ & $\equiv$ & $\equiv$ & $\equiv$ & $\equiv$ & $\equiv$ & $\equiv$ \\
\hline 34 & 378.72 & 62.17 & 8.8 & 179 & 29 & 254.2 \\
\hline$\equiv$ & $\bar{\Xi}$ & $\equiv$ & $\equiv$ & $\bar{\Xi}$ & $\equiv$ & $\equiv$ \\
\hline 100 & 332.9 & 58.16 & 8.8 & 178 & 36 & 224.7 \\
\hline $\bar{x}$ & 320.8 & 59.18 & 9.19 & 163.7 & 21.9 & 253.4 \\
\hline$s$ & 34.45 & 8.58 & 0.69 & 15.43 & 9.27 & 26.99 \\
\hline
\end{tabular}

Table 2. All indicators stándardized values.

\begin{tabular}{|c|c|c|c|c|c|c|}
\hline$n$ & $x_{1}$ & $x_{2}$ & $x_{3}$ & $x_{4}$ & $x_{5}$ & $x_{6}$ \\
\hline 1 & 3.9 & 7.5 & 6.00 & 7.87 & 6.5 & 6.0 \\
\hline 3 & 6.3 & 5.0 & 4.4 & 4.30 & 2.63 & 5.2 \\
\hline $\bar{z}$ & $\bar{z}$ & $\bar{z}$ & $\bar{z}$ & $\bar{z}$ & $\bar{z}$ & $\bar{z}$ \\
\hline 9 & 4.7 & 3.3 & 4.7 & 4.7 & 4.2 & 4.69 \\
\hline $\bar{z}$ & $\bar{z}$ & $\bar{z}$ & $\bar{z}$ & $\bar{z}$ & $\bar{z}$ & $\bar{z}$ \\
\hline 34 & 6.6 & 5.3 & 5.5 & 5.9 & 5.76 & 4.9 \\
\hline $\bar{z}$ & $\bar{z}$ & $\bar{z}$ & $\bar{z}$ & $\bar{z}$ & $\bar{z}$ & $\Xi$ \\
\hline 100 & 5.35 & 4.88 & 5.57 & 5.92 & 6.52 & 6.06 \\
\hline
\end{tabular}

from which d represents center distance of all clustering, absolute value of them represents item $x$ class I indicators center distance; in the case, value $m$ as 6 , according to paper sample $n_{1}$ and I to classify and explain, from which clustering center distance is :

$$
\begin{aligned}
d & =|3.95-4.76|+|7.52-3.39|+\nwarrow+|6.05-4.68| \\
& =12.97
\end{aligned}
$$

Then $n_{1}$ and class II center distance is:

$$
\begin{aligned}
d & =|3.95-6.66|+|7.52-5.37|+\nwarrow+|6.05-4.96| \\
& =10.03
\end{aligned}
$$

So it can conclude the class sample into class II.

After new samples getting into the class, the paper adopts $\left(x_{i}-\overline{x_{i}} \cdot n\right) / n+1$ to define the II all indicators distances, in above examples, it has:
$(3.59+6.69 \times 1) / 1+1=5.3$

$$
(7.52+5.35 \times 1) / 1+1=6.4
$$$$
(6+5.75 \times 1) / 1+1=5.8
$$

By parity of reasoning, it can classify all indicators so that define its center position, through above method, and combine with the paper researched contents; we can get final clustering center results as following Table $\mathbf{3}$ shows:

Do normalization handling with above results according to formula: $x_{i}$ weight $={ }^{t}{ }_{i} / \sum_{t_{i}} \times 100 \%$ to solve all indicators weights values, finally it can get all indicators weights so as to make evaluation, for the paper, it random selects four school girls six indicators to test as following Table 4.

Write above Table 5 into matrix and combine with above gained weights, and then it has: 
Table 3. School girls physique clustering center table.

\begin{tabular}{|c|c|c|c|c|c|c|}
\hline Class & $\begin{array}{c}\mathbf{5 0} \text { Meters } \\
\text { Running }\end{array}$ & $\begin{array}{c}\text { Lung Capacity / } \\
\text { Weight }\end{array}$ & $\begin{array}{c}\text { Weight/ } \\
\text { Height } \times \mathbf{1 0 0 0}\end{array}$ & $\begin{array}{c}\mathbf{8 0 0} \text { Meters } \\
\text { Running }\end{array}$ & $\begin{array}{c}\text { Standing Long } \\
\text { Jump }\end{array}$ \\
\hline \hline Class I & 4.70 & 4.74 & 4.53 & 4.59 & 4.41 & 4.49 \\
\hline Class II & 5.40 & 5.28 & 5.57 & 5.51 & 5.68 & 5.62 \\
\hline
\end{tabular}

Table 4. Two classes' samples mean values test table.

\begin{tabular}{|c|c|c|c|c|c|}
\hline Indicator & Class $\overline{x_{\Pi}}$ & Class $\overline{x_{1}}$ & P value & t value & $S_{\mathrm{II-I}}$ \\
\hline Weight/ height $\times 1000$ & 5.28 & 4.74 & $<.05$ & 2.16 & 0.25 \\
\hline Lung capacity / weight & 4.70 & 5.40 & $<.01$ & 2.69 & 0.26 \\
\hline Sit-up & 5.68 & 4.41 & $<.01$ & 5.52 & 0.23 \\
\hline Standing long jump & 5.62 & 4.49 & $<.01$ & 5.65 & 0.20 \\
\hline 50 meters running & 5.56 & 4.54 & $<.01$ & 6.38 & 0.16 \\
\hline 800 meters running & 5.51 & 4.59 & $<.01$ & 4.00 & 0.23 \\
\hline
\end{tabular}

Table 5. Four school girls physical test result.

\begin{tabular}{|c|c|c|c|c|c|}
\hline Schoolgirl No. & $\mathbf{5 0}$ Meters Running (s) & $\begin{array}{c}1 / 2 \text { Standing Long } \\
\text { Jump (cm) }\end{array}$ & Weight/ Height $\times \mathbf{1 0 0 0}$ & Lung Capacity / weight & Sit-up (Times) \\
\hline \hline 1 & $9.2\left(P_{50}\right)$ & $155\left(P_{40}\right)$ & $310\left(P_{50}\right)$ & $61\left(P_{60}\right)$ & $27\left(P_{50}\right)$ \\
\hline 2 & $9.4\left(P_{40}\right)$ & $155\left(P_{40}\right)$ & $320\left(P_{60}\right)$ & $65\left(P_{40}\right)$ & $68\left(P_{90}\right)$ \\
\hline 3 & $9.0\left(P_{60}\right)$ & $170\left(P_{70}\right)$ & $340\left(P_{80}\right)$ & $63\left(P_{70}\right)$ & $29\left(P_{60}\right)$ \\
\hline 4 & $9.0\left(P_{60}\right)$ & $160\left(P_{70}\right)$ & $300\left(P_{40}\right)$ & $31\left(P_{70}\right)$ \\
\hline
\end{tabular}

$\mathrm{R} \cdot \mathrm{A}=\left[\begin{array}{llllll}60 & 40 & 40 & 40 & 30 & 20 \\ 50 & 60 & 50 & 40 & 50 & 60 \\ 40 & 70 & 60 & 50 & 70 & 80 \\ 80 & 90 & 60 & 70 & 60 & 50\end{array}\right] .\left[\begin{array}{c}0.08 \\ 0.10 \\ 0.24 \\ 0.21 \\ 0.21 \\ 0.15\end{array}\right]$

$=\left[\begin{array}{l}60 \times 0.08+40 \times 0.1+40 \times 0.24+40 \times 0.21+30 \times 0.21+20 \times 0.15 \\ 50 \times 0.08+60 \times 0.1+50 \times 0.24+40 \times 0.21+30 \times 0.21+60 \times 0.15 \\ 40 \times 0.08+70 \times 0.1+60 \times 0.24+50 \times 0.21+70 \times 0.21+80 \times 0.15 \\ 80 \times 0.08+90 \times 0.1+60 \times 0.24+70 \times 0.21+60 \times 0.21+50 \times 0.15\end{array}\right]$

Score above obtained result, then it has:No.1: 36; No.2:49.8; No.3:61.7; No.4:64.6.

\section{CONCLUSION}

The paper's research on Chinese teenager physical health information service system construction targeted at teenagers, it mainly involves objective selection, classification and sorting of selected objectives as well as endowing weights on all indicators. These three aspects are emphasis in the paper, the paper takes normalization processing with multiple indicators according to fuzzy clustering and combining with type test, and then gets fuzzy matrix weight coefficient, and obtains its accurate result by fuzzy aggregation method.

\section{CONFLICT OF INTEREST}

The author confirms that this article content has no conflict of interest.

\section{ACKNOWLEDGEMENTS}

Declared none.

\section{REFERENCES}

[1] J. Cai, B. Fan, and J. Wang, "A survey of residents' satisfaction degree for urban public sport services," Journal of Beijing Sport University, no. 6, 2009.

[2] Y. Chen, and G. Ma, "An empirical study on community sports service residents' satisfaction index model," China Sport Science and Technology, vol. 45, no. 4, 2009. 
[3] Y. He, and M. Xu, "Study on evaluating system of sports consciousness of community residents in southwest cities," Journal of Chengdu Physical Education Institute, vol. 33, no. 2, pp. 43-45, 2007.

[4] B. Liu, S. Hu, H. Xu, and J. Gao, "Indices of the equality of essential public health services in China," Chinese Journal of Health Policy, vol. 2, no. 6, pp. 13-17, 2009.

[5] G. Wang, and E. Zhang, "Construction of the evaluation index system of city community sports-taking shanghai as an example,"
Journal of Chengdu Physical Education Institute, vol. 36, no. 2, 2010.

[6] D. Zhang, and M. Li, "Studies on evaluation index system of public sports facilities development level in china," China Sport Science, vol. 33, no. 4, pp. 3-23, 2013.

[7] J. Zhang, and Y. Wu, "the evaluation index system of extracurricular sports activities in secondary schools in shanghai under the background of "sunshine sports"," Journal of Shanghai Physical Education Institute, no. 6, pp. 80-82, 2012.

Received: June 10, 2015

(C) Yuefeng Zhao; Licensee Bentham Open

This is an open access article licensed under the terms of the (https://creativecommons.org/licenses/by/4.0/legalcode), which permits unrestricted, noncommercial use, distribution and reproduction in any medium, provided the work is properly cited. 